\title{
Conformally-regulated direct integration of the two-loop heptagon remainder
}

\author{
Jacob L. Bourjaily, ${ }^{a, b, c}$ Matthias Volk ${ }^{a}$ and Matt von Hippel ${ }^{a}$ \\ ${ }^{a}$ Niels Bohr International Academy and Discovery Center, Niels Bohr Institute, \\ University of Copenhagen, \\ Blegdamsvej 17, DK-2100, Copenhagen Ø, Denmark \\ ${ }^{b}$ Center for the Fundamental Laws of Nature, Department of Physics, \\ Jefferson Physical Laboratory, Harvard University, \\ Cambridge, MA 02138, U.S.A. \\ ${ }^{c}$ Institute for Gravitation and the Cosmos, Department of Physics, Pennsylvania State University, \\ University Park, PA 16892, U.S.A. \\ E-mail: bourjaily@psu.edu, mvolk@nbi.ku.dk, mvonhippel@nbi.ku.dk
}

\begin{abstract}
We reproduce the two-loop seven-point remainder function in planar, maximally supersymmetric Yang-Mills theory by direct integration of conformally-regulated chiral integrands. The remainder function is obtained as part of the two-loop logarithm of the MHV amplitude, the regularized form of which we compute directly in this scheme. We compare the scheme-dependent anomalous dimensions and related quantities in the conformal regulator with those found for the Higgs regulator.
\end{abstract}

Keywords: Scattering Amplitudes, Supersymmetric Gauge Theory

ARXiv EPRINT: 1912.05690 


\section{Contents}

1 Introduction and overview 1

2 Local integrands for (logarithms of) MHV amplitudes 3

2.1 Specific contributions to the seven-point logarithm 6

3 Feynman parameterization and direct integration $\quad 6$

3.1 (Cluster) coordinate charts for heptagon integrals $\quad 7$

$\begin{array}{ll}3.2 & \text { Divide and conquer: parametric integration via various pathways }\end{array}$

3.3 Refining the results of integration (removing spurious letters) 8

4 The two-loop heptagon remainder function 10

4.1 Exempli gratia: Higgs-regulated (logarithms of) MHV amplitudes 10

$\begin{array}{ll}4.2 \text { Conformally-regulated (logarithms of) MHV amplitudes } & 11\end{array}$

$\begin{array}{lll}\text { 4.3 Symbology and the alphabets of individual integral contributions } & 12\end{array}$

5 Discussion 13

\section{Introduction and overview}

The study of scattering amplitudes in recent decades has led to tremendous advances in both our understanding of quantum field theory and also our technical progress in computing the predictions made for experiment. Much of this progress can be attributed to the remarkable (and still surprising) simplicity of massless quantum field theories in four dimensions. Any such theory turns out to possess a connection to Grassmannian geometry [1-4] which has led to novel applications and greater understanding of perturbative amplitudes for an expanding class of quantum theories. This is true despite the subtlety involved in even defining the $S$-matrix for massless field theories! (But see $[5,6]$ for recent progress on this problem.)

Many of the difficulties of working with massless quantum field theories can be postponed by focusing on loop integrands ('the sum of Feynman diagrams'). At the integrand level, there are several new and extremely powerful frameworks for expressing perturbative scattering amplitudes of an increasingly general class of theories. These tools include all-loop recursion relations [7, 8], bootstrap methods [9-11], $Q$-cuts [12], and the broad reach of generalized [13-22] and prescriptive [23-29] unitarity. It remains to be seen, however, how much of the simplicity of integrands can survive loop integration. Considering the extent to which the simplicity at the integrand-level arises specifically for theories of massless particles in exactly four dimensions, and that it is precisely these features that are responsible for infrared divergences whose regularization necessarily spoils them, it 
would not be surprising if much of this extra structure was lost to the infrared. Indeed, it would be reasonable to be skeptical that anything remarkable would be found for the actual infrared-safe quantities in which we are ultimately interested.

To test whether or not any of the niceness of amplitudes at the integrand-level survives the wrath and fury (the infrared regularization) of loop integration, it would be reasonable to simply 'shut up and calculate' - by any means necessary - and see what emerges in the '[theoretical] data', so to speak. Of course, this will always be easier to accomplish for especially simple quantum field theories such as maximally supersymmetric $(\mathcal{N}=4)$ YangMills ('sYM') in the planar limit, for which the greatest computational leverage exists (largely due to this theory's special properties [30-35]).

There is a now-quite-famous example which illustrates what can be discovered through such a 'compute first, understand later' strategy. It involves one of the simplest nonconstant and non-trivial infrared-safe quantities in planar sYM: the (BDS) remainder function for six particles at two-loop order. This quantity was determined through truly heroic efforts, first numerically [20] and then analytically [36] — in both cases, starting from an integrand-level expression obtained using unitarity-based methods; then regulating; then integrating. Within months of the publication of the analytic result, however, breathtaking simplicity was indeed found: the 18-page sum of hyperlogarithms in [36] could be written in a single line [37]!

The ideas that led to the discovery of this simplicity would lead to a watershed of new and powerful techniques developed hand-in-hand with even greater evidence of simplicity surviving regularization and loop integration. Today, this particular quantity - the sixparticle remainder function in planar sYM — is known to seven(!) loops; and the sevenparticle remainder is known (at least at 'symbol-level') to four loops [38-49]. Interestingly, after the two-loop result was found 'the old fashioned way' in [36] — namely, by integrating Feynman integrands - all subsequent results were obtained using methods that made no reference to loop integrands or loop integration whatsoever! While these ideas have more recently been applied to non-planar amplitudes in supersymmetric theories $[50,51]$ and more broadly [51-60], they suffer from several fundamental limitations in applicability - in multiplicity, in the understanding (and simplicity) of the kinds of transcendental functions that arise in perturbation theory (including those described in e.g. [61]) — that prevent these ideas from rewriting the methods taught in textbooks, say.

One of the key motivations for our present work is the question of how much simplicity of loop integrands can be preserved through loop integration and regularization. Specifically, how can this bridge be crossed by direct and general methods - without reference to any ansatz about the kinds of functions that may arise in particular cases. A key source of hope that a more direct (and therefore general) connection between the remarkable integrands for amplitudes in planar sYM [24-26] and the simple expressions that we now expect to find for infrared-safe quantities is the is the existence of the regulator introduced in [24], which allows infrared divergences to be regulated without breaking (dual-)conformal invariance. Another critical source of optimism is the recent renaissance in direct-integration technology for Feynman-parametric integrands [62-64] (see also [65, 66]). 
In this work, we test the robustness of this emerging bridge from integrands to integrals in the highly non-trivial case of the seven-point remainder function at two loops. This quantity was first determined at symbol-level in [67] (see also [68, 69]), and later upgraded to a function-level result in [70]. Here, we start from the chiral integrand representation for the logarithm of the amplitude given in [23], use the conformal regulator of [24], Feynman-parameterize these terms according to [71], and integrate each piece using the technology of [62-64]. The result is a novel (if not superior) representation of the two-loop remainder function, and a proof of concept that such a strategy can work. As a bonus, by combining this result with that of [71] for six particles, we are able to determine all of the scheme-dependent parts of the two-loop MHV-amplitude logarithm in the conformal regularization scheme.

This work is organized as follows. We start in section 2 with a review of the local integrands necessary for MHV amplitudes and their logarithms in planar sYM at two-loops and how these integrands can be regulated while preserving dual-conformal invariance. In section 3 we discuss how we can directly integrate each of the integrands needed for the seven-particle logarithm, resulting in a representation in terms of explicit hyperlogarithmic functions. Our main results regarding the heptagon remainder function are described in section 4, where we determine the scheme-dependent parts of the logarithm of MHV amplitudes in the conformal regularization scheme and compare these with what is found for the Higgs regulator.

Available as supplementary material attached to this paper, we have prepared the supplementary file heptagon_logarithm_seed_data.m. This file contains: Feynmanparametric integrands for the five (cyclic) seeds which generate the seven-point logarithm at two loops; analytic expressions for each seed integral — given in terms of Goncharov hyperlogarithms - obtained via direct integration; details regarding the novel alphabets that arise for these integrals; and reference details regarding how our coordinates related to those used by [70] in their representation of the two-loop heptagon remainder function.

\section{Local integrands for (logarithms of) MHV amplitudes}

In this section, we give a rapid review of the representation (in terms of local Feynman integrals) of $\mathrm{MHV}$ amplitudes and their logarithms at two loops in the planar limit of sYM. In [7] (see also the earlier work [20, 72, 73]), it was guessed (and checked) that the $n$-particle MHV amplitude integrand could be represented as ${ }^{1}$

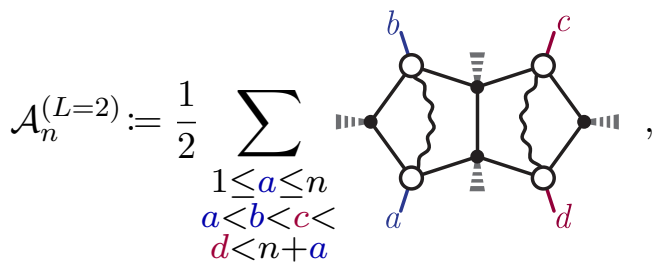

\footnotetext{
${ }^{1}$ Notice that we have dropped the typical notation indicating $\mathrm{N}^{(\mathbf{k}=0)} \mathrm{MHV}$ degree in ' $\mathcal{A}_{n}^{(L)}$, , as no other helicity sectors will be considered in this work.
} 
where the double-pentagons, herein ' $\Omega[(a, b),(c, d)]$ ', have precise loop-dependent numerators (indicated by the wavy-lines in the figure) expressed in terms of momentum twistors [74]:

$$
=\frac{\left\langle\left(\ell_{1}\right)(a-1 a a+1) \cap(b-1 b b+1)\right\rangle\langle b a d c\rangle\left\langle\left(\ell_{2}\right)(c-1 c c+1) \cap(d-1 d d+1)\right\rangle}{\left(\ell_{1} \mid a\right)\left(\ell_{1} \mid a+1\right)\left(\ell_{1} \mid b\right)\left(\ell_{1} \mid b+1\right)\left(\ell_{1} \mid \ell_{2}\right)\left(\ell_{2} \mid c\right)\left(\ell_{2} \mid c+1\right)\left(\ell_{2} \mid d\right)\left(\ell_{2} \mid d+1\right)}
$$

As usual, we are using the notations $(a \mid b):=\left(x_{a}-x_{b}\right)^{2}$ where $x_{a}$ are the dual coordinates related to the momenta through $p_{a}=: x_{a+1}-x_{a}$, and $\langle a b c d\rangle:=\operatorname{det}\left(z_{a}, z_{b}, z_{c}, z_{d}\right)$ for the ordinary four-brackets of momentum twistors.

We should clarify that the factor of ' $1 / 2$ ' appearing in $(2.1)$ is really a symmetry factor: it accounts for the fact that the summand includes each contribution exactly twice - provided we view the integrand in (2.2) as being (implicitly) symmetrized with respect to $\ell_{1} \leftrightarrow \ell_{2}$; in particular, this factor of $1 / 2$ could be dispensed by an instruction to 'delete duplicates' from the r.h.s. (something often left implicit in the relevant literature). As $\Omega[(a, b),(c, d)]$ and $\Omega[(c, d),(a, b)]$ are identical upon integration, we consider them equivalent (a.k.a. 'duplicates') - a potential source of confusion below, for which we apologize.

Notice that the definition of $\Omega[(a, b),(c, d)]$ depends on up to twelve momentum twistors

$$
\left\{z_{a-1}, z_{a}, z_{a+1}\right\} \cup\left\{z_{b-1}, z_{b}, z_{b+1}\right\} \cup\left\{z_{c-1}, z_{c}, z_{c+1}\right\} \cup\left\{z_{d-1}, z_{d}, z_{d+1}\right\}
$$

with cyclic labeling understood. Especially for low multiplicity, these indices can overlap considerably. When it is necessary to disambiguate the multiplicity $n$, implicit in the definition (2.2) above, we will signify this by writing ' $\Omega^{(n)}[(a, b),(c, d)]$ '.

Shortly after the formula (2.1) appeared in [7], a similar expression was derived in [23] for the four-dimensional integrand of the two-loop logarithm of the MHV amplitude,

$$
\log \left(\mathcal{A}_{n}\right)^{(L=2)}=\mathcal{A}_{n}^{(L=2)}-\frac{1}{2}\left(\mathcal{A}_{n}^{(L=1)}\right)^{2}=-\frac{1}{4} \sum_{\substack{1 \leq a<n \\ a<c<b<\\ d<n+a}} \Omega[(a, b),(c, d)]
$$

(As before, the factor of ' $1 / 4$ ' above is merely a symmetry factor: the appropriate prefactor would be 1 times each term in the summand without duplication.) Notice that the summand in (2.4) now excludes the possibility that $a+1=b$ and - more importantly - the summand requires that $c \in\{a+1, \ldots, b-1\}$.

It is instructive to see a few instances of equation (2.4). Without symmetry factors, but being explicit about the fact that cyclic seeds should be summed only without duplication, and being very careful about which cyclic seeds necessitate clarification about when multiplicity matters, the two-loop logarithms of MHV amplitudes for 4-8 particles are as 
follows:

$$
\begin{aligned}
& \log \left(\mathcal{A}_{4}\right)^{(2)}=-\left[\Omega^{(4)}[(2,4),(3,1)]+\underset{\text { (no dupl.) }}{\text { cyclic }_{4}}\right]=-\Omega^{(4)}[(2,4),(3,1)], \\
& \log \left(\mathcal{A}_{5}\right)^{(2)}=-\left[\Omega^{(5)}[(2,4),(3,5)]+\underset{(\text { no dupl. })}{\text { cyclic }_{5}}\right]=-\left[\Omega^{(5)}[(2,4),(3,5)]+\text { cyclic }_{5}\right] \text {, } \\
& \log \left(\mathcal{A}_{6}\right)^{(2)}=-\left[\Omega[(2,4),(3,5)]+\Omega^{(6)}[(2,4),(3,6)]+\Omega^{(6)}[(2,5),(3,6)]+\underset{\text { (no dupl. })}{\text { cyclic }_{6}}\right], \\
& \log \left(\mathcal{A}_{7}\right)^{(2)}=-[\Omega[(2,4),(3,5)]+\Omega[(2,4),(3,6)]+\Omega[(2,5),(3,6)] \\
& \left.+\Omega^{(7)}[(2,4),(3,7)]+\Omega^{(7)}[(2,5),(3,7)]+\underset{(\text { no dupl. })}{\operatorname{cyclic}_{7}}\right], \\
& \log \left(\mathcal{A}_{8}\right)^{(2)}=-[\Omega[(2,4),(3,5)]+\Omega[(2,4),(3,6)]+\Omega[(2,5),(3,6)] \\
& +\Omega[(2,4),(3,7)]+\Omega[(2,5),(3,7)]+\Omega[(2,6),(3,7)]+\Omega^{(8)}[(2,4),(3,8)] \\
& \left.+\Omega^{(8)}[(2,5),(3,8)]+\Omega^{(8)}[(2,5),(4,8)]+\Omega^{(8)}[(2,6),(4,8)]+\underset{\text { (no dupl. })}{\text { cyclic }_{8}}\right] .
\end{aligned}
$$

There are a couple of things to notice about these representations. First, observe that for more than six particles the majority of cyclic seeds can be chosen to be independent of $n$; therefore, these contributions remain unchanged beyond some threshold multiplicity. The second thing to notice is that it is fairly easy to organize contributions according to their degrees of infrared divergence: ${ }^{2}$

$$
\begin{aligned}
& \log ^{2} \text {-divergent: } \Omega[(2,4),(3,5)] \text { only, } \\
& \log ^{1} \text {-divergent: } \Omega[(2,4),(3, b)] \text { for } b>5,
\end{aligned}
$$

with all other integrals finite. In particular, notice that the only cyclic seed with a $\log ^{2}$ divergence is $\Omega[(2,4),(3,5)]$ and that this integral is $n$-independent once it is evaluated for any $n \geq 6$. We will return to the consequences of this fact momentarily.

To regulate these divergences, we employ the so-called 'dual-conformal' regularization scheme introduced in [24], wherein each (massless) external particle is taken off the lightcone by an amount proportional to the conformally-invariant parameter denoted ' $\delta$ ' according to

$$
p_{a}^{2} \mapsto p_{a}^{2}+\delta \frac{\left(p_{a-1}+p_{a}\right)^{2}\left(p_{a}+p_{a+1}\right)^{2}}{\left(p_{a-1}+p_{a}+p_{a+1}\right)^{2}}=(a \mid a+1)+\delta \frac{(a-1 \mid a+1)(a \mid a+2)}{(a-1 \mid a+2)} .
$$

(There is an alternative definition of this regulator expressed in terms of dual-momentum coordinates - where each dual coordinate $x_{a}$ is shifted by a small amount in the direction of its cyclic neighbor, $x_{a+1}$; these two definitions are not identical for finite $\delta$, but they result in regulated integrals equivalent to $\mathcal{O}(\delta)$.)

\footnotetext{
${ }^{2}$ In dimensional regularization, ' $\log ^{k}$-divergent' should be understood as ' $1 / \epsilon^{k}$-divergent'.
} 


\subsection{Specific contributions to the seven-point logarithm}

As seven particles is the primary example of interest to us here, it is worthwhile to give the five cyclic generators in (2.8) individual names. Let us therefore define

$$
\begin{array}{lll}
\mathcal{I}_{1}:=\Omega[(2,4),(3,5)], & \mathcal{I}_{2}:=\Omega[(2,4),(3,6)], & \mathcal{I}_{3}:=\Omega[(2,5),(3,6)], \\
\mathcal{I}_{4}:=\Omega^{(7)}[(2,4),(3,7)], & \mathcal{I}_{5}:=\Omega^{(7)}[(2,5),(3,7)] . &
\end{array}
$$

Notice that from our discussion above, only $\mathcal{I}_{1}$ will be $\log ^{2}$-divergent in the infrared upon integration, while $\left\{\mathcal{I}_{2}, \mathcal{I}_{4}\right\}$ will be $\log ^{1}$-divergent; the two seeds $\left\{\mathcal{I}_{3}, \mathcal{I}_{5}\right\}$ are infrared finite, and therefore do not require any regularization.

We will discuss how each of the contributions (2.12) can be evaluated in the following section. But already now we can observe an important consequence of the fact that $\mathcal{I}_{1}$ depends exclusively on momentum twistors $\left\{z_{1}, \ldots, z_{6}\right\}$ : its evaluation will be the same for seven particles as it was for six. More specifically, $\mathcal{I}_{1}$ is essentially identical to what was computed (as part of what was called ' $\mathcal{I}_{15}$ ') in [71]

$$
\begin{aligned}
I_{1} & =\int d^{4} \ell_{1} d^{4} \ell_{2} \mathcal{I}_{1} \\
& =\frac{1}{4}\left[2 \zeta_{2} \log ^{2}(\delta)+6 \zeta_{3}[\log (\delta)+1]-\zeta_{2}^{2}-2 \zeta_{2} G_{0,1}(1-w)+G_{0,0,0,1}(1-w)-G_{0,1,0,1}(1-w)\right]
\end{aligned}
$$

where

$$
w:=\frac{(3 \mid 5)(6 \mid 2)}{(3 \mid 6)(5 \mid 2)}=\frac{\langle 2345\rangle\langle 5612\rangle}{\langle 2356\rangle\langle 4512\rangle} .
$$

Notice that we are reserving calligraphic symbols to denote integrands and italic symbols to indicate integrals.

As $\mathcal{I}_{1}$ is the only cyclic seed with a $\log ^{2}$-divergence for arbitrary $n$, it is wholly responsible for the leading divergence of the logarithm of MHV amplitudes at two loops. The coefficient of this divergence is related to the (scheme independent) cusp anomalous dimension, and the attentive reader can already see that (2.13) captures the right behavior. We will see this in detail in section 4 below; but before we do, it is worthwhile to describe how the other seven-point seeds have been evaluated analytically.

\section{Feynman parameterization and direct integration}

Following the strategy described in [71], it is straightforward to Feynman-parameterize and regulate each of the contributions (2.12). For each of the double-pentagon integrals, this will result in a rational, five-dimensional parametric integral representation of the form ${ }^{3}$

$$
I_{i}:=\int_{0}^{\infty}\left[d^{3} \vec{\alpha}\right] d^{2} \vec{\beta} \mathcal{I}_{i}\left(\vec{\alpha}, \vec{\beta} ;\left\{z_{1}, \ldots, z_{7}\right\}, \delta\right)
$$

\footnotetext{
${ }^{3}$ We hope the reader will forgive our abuse of notation in using ' $\mathcal{I}_{i}$ ' to denote both the loop-momentumspace and Feynman-parametric integrands.
} 
In the integral above, $\left[d^{3} \vec{\alpha}\right]:=d^{4} \vec{\alpha} \delta\left(\alpha_{j}-1\right)$ (for any $j$ ) represents a projective, 3-dimensional volume-form; while the $\beta$ integrations are not taken to be projective. This distinction is largely irrelevant due to the Cheng-Wu theorem [75]; but it reflects the way in which the parametric representations were derived via [71], and we find it useful to keep this information. In the supplementary material, we provide a parametric representation of each of the seven-point integrals in (2.12).

\section{1 (Cluster) coordinate charts for heptagon integrals}

In (2.2) we have given the formula for $\Omega[(a, b),(c, d)]$ in terms of momentum twistors $z_{a} \in \mathbb{P}^{3}$ for $a=1, \ldots, n$ that parameterize the kinematic space of $n$ massless particles. As described in detail in [65] a momentum-twistor parameterization is preferred over one expressed in terms of dual-momentum $x$-coordinates, as twistor space immediately provides us with an integrand that is rational in terms of an independent set of conformal variables.

It turns out that the default cluster coordinates on $G_{+}(4, n)$ of the Mathematica package positroids [76] provide a very convenient chart for our present purposes. For a more detailed discussion of these coordinates we again refer the reader to [65]. For seven points, we can think of these coordinates as parameterizing seven momentum twistors $Z=:\left(z_{1} \cdots z_{7}\right)$ according to

$$
Z\left(\left\{e_{a}^{i}\right\}\right):=\left(\begin{array}{ccccccc}
1 & 1+e_{6}^{3}+e_{7}^{3} & e_{6}^{3}+\left(1+e_{6}^{2}\right) e_{7}^{3} & e_{6}^{2} e_{7}^{3} & 0 & 0 & 0 \\
0 & 1 & 1+e_{6}^{2}+e_{7}^{2} & e_{6}^{2}+\left(1+e_{6}^{1}\right) e_{7}^{2} & e_{6}^{1} e_{7}^{2} & 0 & 0 \\
0 & 0 & 1 & 1+e_{6}^{1}+e_{7}^{1} & e_{6}^{1}+e_{7}^{1} & e_{7}^{1} & 0 \\
0 & 0 & 0 & 1 & 1 & 1 & 1
\end{array}\right)
$$

or, if viewed as coordinates (maps from $G_{+}(4,7) \mapsto \mathbb{R}^{6}$ ), the parameters $\left\{e_{a}^{i}\right\}$ correspond to the conformal cross-ratios

$$
\begin{array}{lll}
e_{6}^{1}:=\frac{\langle 1234\rangle\langle 1256\rangle}{\langle 1236\rangle\langle 1245\rangle}, & e_{6}^{2}:=\frac{\langle 1235\rangle\langle 1456\rangle}{\langle 1256\rangle\langle 1345\rangle}, & e_{6}^{3}:=\frac{\langle 1245\rangle\langle 3456\rangle}{\langle 1456\rangle\langle 2345\rangle}, \\
e_{7}^{1}:=\frac{\langle 1234\rangle\langle 1235\rangle\langle 1267\rangle}{\langle 1236\rangle\langle 1237\rangle\langle 1245\rangle}, & e_{7}^{2}:=\frac{\langle 1236\rangle\langle 1245\rangle\langle 1567\rangle}{\langle 1256\rangle\langle 1267\rangle\langle 1345\rangle}, & e_{7}^{3}:=\frac{\langle 1256\rangle\langle 1345\rangle\langle 4567\rangle}{\langle 1456\rangle\langle 1567\rangle\langle 2345\rangle} .
\end{array}
$$

\subsection{Divide and conquer: parametric integration via various pathways}

The seed integrands expressed in this way can be integrated in terms of hyperlogarithms [77-79] (e.g. using HyperInt [63, 64]) if there exists an order of the integration variables in which the integrand is linearly reducible. Naïvely, however, this turns out not to be the case for any of the integrals at hand: all require some minor 'tricks' of integration analogous to those discussed in, for example, [65, 66, 71, 78, 80].

Among the integration techniques required are those that allow us to extract the leading terms in the limit of $\delta \rightarrow 0^{+}$(for the integrals which require regularization). We were able to effectively use the methods discussed in [71]; we refer the reader to appendix B.1 and the ancillary files of that work for a more thorough explanation and illustrative examples.

Of the two infrared finite integral seeds, only $\mathcal{I}_{5}$ required mild cleverness to integrate directly. For this integral, a strategy which started along similar lines to that described in [66] worked quite well. Specifically, starting from the Feynman-parametric integrand 
representation of the form (3.1) (provided in the supplementary material), we found that the integrals over $\alpha_{2}, \beta_{1}$, and $\beta_{2}$ could each be performed rationally - i.e. without introducing any algebraic dependence on the remaining integration variables in the arguments of the hyperlogarithms or their prefactors.

The (projective) two-fold parametric representation of $\mathcal{I}_{5}$ obtained in this way suffers from a mild problem all-too familiar in these examples: integration in any one of the remaining variables would result in some terms with a square root depending (quadratically) on the final integration variable. Such an obstruction is easy to overcome by changing variables (Euler substitution) as described in e.g. [78, 80]. But a better pathway to integration turns out to exist: the individual terms of the two-fold parametric representation of $\mathcal{I}_{5}$ can be divided into groups which separately avoid this issue with respect to integration in $\alpha_{4}$ or $\alpha_{1}$. This results in a final expression with fewer 'spurious' algebraic symbol letters to be discussed in the next section.

\subsection{Refining the results of integration (removing spurious letters)}

Following the strategies discussed above, it was fairly easy to obtain hyperlogarithmic (regulated, if necessary) expressions for integrals $\left\{I_{1}, \ldots, I_{4}\right\}$; but integration of $\mathcal{I}_{5}$ required some cleverness, resulting in a representation of $I_{5}$ that is considerably more complicated in two key aspects: first, the representation we obtained for $I_{5}$ was not manifestly pure in the sense of $[23,81]$ - namely, it was expressed as a sum of hyperlogarithms with nonconstant (algebraic) coefficients; and second, it was expressed in terms of hyperlogarithms with many (suspected to be 'spurious') algebraic branch points. Let us discuss each of these complications in turn.

The first complication, regarding the non-manifest 'purity' of $I_{5}$ turns out to be straightforward to deal with. First, we should clarify why we expected $I_{5}$ to be pure despite its representation. Although the conformal regulator is known to spoil an integrand's purity (see the discussion in [71]), we strongly expect the logarithm of the amplitude (the cyclic sum of all seeds) to be pure; as $\left\{I_{1}, \ldots, I_{4}\right\}$ were individually pure, it would require considerable magic for impurities of $I_{5}$ to cancel amongst themselves in the cyclic sum.

Setting aside our expectations about $I_{5}$ 's purity, it turns out to be fairly easy to test whether or not any non-manifestly pure sum of hyperlogarithms is in fact pure. Suppose that some non-manifestly pure sum of hyperlogarithms $I\left(\left\{e_{a}^{i}\right\}\right)$ depending on parameters $\left\{e_{a}^{i}\right\}$ is in fact pure; then we should be able to re-express it in terms of some basis of hyperlogarithms $\left\{G_{\beta}\right\}$ :

$$
I\left(\left\{e_{a}^{i}\right\}\right):=\sum_{\alpha} R_{\alpha}\left(\left\{e_{a}^{i}\right\}\right) G_{\alpha}\left(\left\{e_{a}^{i}\right\}\right) \Rightarrow \sum_{\beta} c_{\beta} G_{\beta}\left(\left\{e_{a}^{i}\right\}\right)
$$

where $R_{\alpha}$ are rational(/algebraic)-function prefactors, $c_{\beta}$ are constants, and $G_{\alpha}, G_{\beta}$ multiple polylogarithms. In order for (3.4) to be true, there would need to be some relations among the functions $G_{\alpha}$. Crucially, any such relations would necessarily be linear and have constant coefficients - as all relations between multiple polylogarithms are expected to preserve transcendental weight and not involve any rational functions of their arguments. 
Now suppose we were to Taylor-expand each coefficient $R_{\alpha}$ in (3.4) around some point $\widehat{e_{a}^{i}}$ where all the $R_{\alpha}$ 's are non-singular. Then we would have

$$
\sum_{\alpha}\left[\sum_{j=0}^{\infty} R_{\alpha}^{(j)}\left(e_{a}^{i}-\widehat{e_{a}^{i}}\right)^{j}\right] G_{\alpha}\left(\left\{e_{a}^{i}\right\}\right)=\sum_{\beta} c_{\beta} G_{\beta}\left(\left\{e_{a}^{i}\right\}\right) .
$$

Since all purported relations among the $\left\{G_{\alpha}\right\}$ are linear, this requires that the identity (3.5) holds for each term in the Taylor series separately. In particular, it must hold at leading order. Moreover, as each $R_{\alpha}^{(0)}$ is just some constant, this term in the left-hand side of (3.5) is itself pure.

The above discussion shows that when an integral is in fact pure, any representation like that on the l.h.s. of (3.4) can be replaced by series-expanding each coefficient to leading order around any non-singular point, resulting in a manifestly pure representation. To test whether or not an integral is in fact pure, we can simply evaluate both ends of this algorithm numerically and check that they agree. For $I_{5}$ we have checked in this way that it in fact pure, and have provided a manifestly pure representation (obtained in this way) in the supplementary material.

The second complication about the representation of $I_{5}$ obtained in the manner described above (namely, divide and conquer) is that this method has a tendency to introduce 'spurious' branch points among terms (which cancel between the divided pieces). When these spurious branch points are not rational in the variables $\left\{e_{a}^{i}\right\}$, we know of no general strategy to canonically eliminate them (as we would by choosing a fibration basis, for example, had they been rational). Removing a dependence on spurious square roots from polylogarithmic expressions is in general a difficult problem, and one we will not attempt to solve here.

Although we have not found a representation for $I_{5}$ free of spurious square-root branch points, we are able to confirm that all non-rational branch points are indeed spurious. To do this, we first compute the symbol $[37,82]$ of $I_{5}$, resulting in an alphabet of 85 letters, 22 of which involve square roots. These algebraic letters appear in pairs of the form $\rho \pm \sqrt{\sigma}$, which can be multiplied to generate root-free letters, leaving us with only 11 algebraic letters to analyze.

These 11 spurious letters are not all independent. Unlike for symbols involving only rational letters, merely factoring square-root letters is not enough to trivialize all identities due to the absence of a unique factorization domain (for further discussion, see [66]). Here we do not need to make use of the more mathematically sophisticated methods [66]. Instead, we simply observe that products of pairs of our remaining eleven letters can yield letters that appear elsewhere in the symbol. By taking into account all such pairings, we find six relations between the 11 letters, and imposing these results in a manifestly rational symbol. This rationalized symbol for $I_{5}$ can now be viewed as canonical, and consists of 47 letters (functions of momentum twistor cross-ratios).

From the symbol of $I_{5}$, it would be possible to reconstruct a rational, hyperlogarithmic representation - using essentially the same techniques by which the two-loop heptagon remainder function was first obtained in [70] from its symbol, which in turn was first 
computed in [67] (see also $[68,69]$ ). We choose not to pursue this for $I_{5}$ because functional reconstruction is not our goal here. Rather, we are interested in how far we may push direct integration of local integrals. One can easily check that the representation we give for $I_{5}$ - despite its spurious letters — perfectly matches Monte Carlo integration.

\section{The two-loop heptagon remainder function}

We are now ready to describe the results of our analysis - to discover the form of the (all-orders) relationship between the logarithm of the MHV amplitude and the so-called 'BDS' remainder function [20] in the conformal regularization scheme. Both for the sake of comparison and in order to introduce some useful notation, let us first pause to review the form of this relationship in the so-called 'Higgs' regularization scheme described in [83, 84].

\subsection{Exempli gratia: Higgs-regulated (logarithms of) MHV amplitudes}

At leading order in the coupling $a:=g^{2} N_{c} /\left(8 \pi^{2}\right)$, the MHV amplitude (divided by the tree) and its logarithm are identical (in any regularization scheme 'reg.'):

$$
\log \left(A_{n, \text { reg. }}\right)=\sum_{\ell=1}^{\infty} a^{\ell} \log \left(A_{n, \text { reg. }}\right)^{(\ell)}=a A_{n, \text { reg. }}^{(1)}+a^{2}\left[A_{n, \text { reg. }}^{(2)}-\frac{1}{2}\left(A_{n, \text { reg. }}^{(1)}\right)^{2}\right]+\mathcal{O}\left(a^{3}\right) .
$$

(Recall our convention that calligraphic symbols such as $\mathcal{A}$ denote integrands while italic symbols such as $A$ denote integrals.) As such, it is useful to first review the form of the one-loop amplitude in the relevant regularization scheme.

For the Higgs regulator described in [83, 84], one loop MHV amplitudes take the form

$$
A_{n, \text { Higgs }}^{(1)}=:-\frac{1}{4}\left[\sum_{a=1}^{n} \log ^{2}\left(\frac{m_{a}^{2}}{(a \mid a+2)}\right)\right]+F_{n, \text { Higgs }}^{(1)}+\mathcal{O}\left(m_{a}^{2}\right),
$$

where $F_{n \text {,Higgs }}^{(1)}$ is the so-called ${ }^{4}$ 'finite part' of the one-loop amplitude in this scheme, and where we have added an index ' $a$ ' $\in[n]$ to distinguish between the various internal masses $m_{a}^{2}$ (which are typically taken to be the same). Notice that we are using dual-momentum notation where $(a \mid b):=\left(x_{a}-x_{b}\right)^{2}:=\left(p_{a}+\ldots+p_{b-1}\right)^{2}$. It is worthwhile to consider the direction along the Higgs branch where these masses scale according to

$$
m_{a}^{2} \mapsto \delta \frac{(a-1 \mid a+1)(a \mid a+2)}{(a-1 \mid a+2)}
$$

under which

$$
A_{n, \text { Higgs }}^{(1)} \underset{(4.3)}{\longrightarrow}-\frac{1}{4}\left[n \log ^{2}(\delta)+\log (\delta) \log \left(w_{1} \cdots w_{n}\right)+\sum_{a=1}^{n} \log ^{2}\left(\frac{(a \mid a+2)}{(a \mid a+3)}\right)\right]+F_{n, \text { Higgs }}^{(1)}+\mathcal{O}(\delta),
$$

where the cross-ratio $w_{a}$ is given by

$$
w_{a}:=\frac{(a \mid a+2)(a+3 \mid a+5)}{(a \mid a+3)(a+2 \mid a+5)} .
$$

\footnotetext{
${ }^{4}$ It is so-called despite the fact that the leading term of (4.2) includes parts finite as $m_{a}^{2} \rightarrow 0$.
} 
This is extremely similar to the form of the one loop amplitude in the conformal regularization scheme. Before we get to that, however, let us first recall a few more facts about the Higgs regulator and the form that the logarithm (4.1) takes in this scheme.

In [84], the all-order form of the logarithm (4.1) was represented according to the BDS ansatz [85] as

$$
\begin{aligned}
& \log \left(A_{n, \text { Higgs }}\right)=-\frac{\gamma_{c}(a)}{16} A_{n, \text { Higgs }}^{(1)}+\frac{\widetilde{\mathcal{G}}_{0}(a)}{2} \sum_{a=1}^{n} \log \left(\frac{m_{a}^{2}}{(a \mid a+2)}\right)+n \widetilde{f}(a)+\widetilde{C}(a)+R_{n}(a) \\
& \underset{(4.3)}{\longmapsto}-\frac{\gamma_{c}(a)}{16} A_{n, \text { Higgs }}^{(1)}+\frac{\widetilde{\mathcal{G}}_{0}(a)}{2}\left[n \log (\delta)+\frac{1}{2} \log \left(w_{1} \cdots w_{n}\right)\right] \\
&+n \widetilde{f}(a)+\widetilde{C}(a)+R_{n}(a)
\end{aligned}
$$

where $\gamma_{c}(a)$ is the (scheme-independent) cusp anomalous dimension $[86,87]$

$$
\gamma_{c}(a)=\sum_{\ell=1}^{\infty} a^{\ell} \gamma_{c}^{(\ell)}=4 a-4 \zeta_{2} a^{2}+22 \zeta_{4} a^{3}-\left(24 \zeta_{2}^{3}+4 \zeta_{3}^{2}+2 \zeta_{2} \zeta_{4}+\zeta_{6}\right) a^{4}+\mathcal{O}\left(a^{5}\right),
$$

$\widetilde{\mathcal{G}}_{0}(a), \widetilde{f}(a), \widetilde{C}(a)$ are scheme-dependent functions of the coupling and $R_{n}(a)$ is the remainder function [20]. In the Higgs regularization scheme these functions were determined by $[83,84]$ to be

$$
\widetilde{\mathcal{G}}_{0}(a)=-\zeta_{3} a^{2}+\mathcal{O}\left(a^{3}\right), \quad \widetilde{f}(a)=\frac{1}{2} \zeta_{4} a^{2}+\mathcal{O}\left(a^{3}\right), \quad \widetilde{C}(a)=-\frac{5}{4} \zeta_{4} a^{2}+\mathcal{O}\left(a^{3}\right),
$$

at two-loop order. (See e.g. [88, 89] for more recent, higher-order results.)

With this comparison in mind, let us now return to the main purpose of this work and describe the form the logarithm takes for the conformal regularization scheme.

\subsection{Conformally-regulated (logarithms of) MHV amplitudes}

Using the conformal regulator described in [24] the divergences of one-loop amplitudes take a form strikingly similar to that of (4.4). In this scheme, the $n$-point MHV amplitude is given by ${ }^{5}$

$$
A_{n, \mathrm{DCI}}^{(1)}:=-\frac{1}{2}\left[n \log ^{2}(\delta)+\log (\delta) \log \left(w_{1} \cdots w_{n}\right)+n \zeta_{2}+F_{n, \mathrm{DCI}}^{(1)}\right]+\mathcal{O}(\delta),
$$

where the cross-ratios $w_{a}$ are the same as those defined in (4.5) and

$$
F_{n, \mathrm{DCI}}^{(1)}=\left[\sum_{b=4}^{\lfloor n / 2\rfloor+1} \operatorname{Li}_{2}\left(1-u_{1, b}\right)+\frac{1}{2} \log \left(u_{1, b}\right) \log \left(v_{1, b}\right)\right]+\underset{\text { (delete duplicates) }}{\text { cyclic }_{n}}
$$

where the cross-ratios $u_{a, b}$ and $v_{a, b}$ are given by

$$
u_{a, b}:=\frac{(a+1 \mid b)(b+1 \mid a)}{(a+1 \mid b+1)(b \mid a)}, \quad v_{a, b}:=\frac{(a-1 \mid a+1)(a \mid a+2)(b-1 \mid b+1)(b \mid b+2)}{(a-1 \mid a+2)(a \mid b)(b-1 \mid b+2)(b+1 \mid a+1)} .
$$

\footnotetext{
${ }^{5}$ We have added a factor of $1 / 2$ relative to [24] to match conventions for the coupling $a$.
} 
In terms of the regulated amplitude at one loop (4.9), it was suggested in [71] that the conformally regulated logarithm (4.1) would take the form

$$
\begin{aligned}
\log \left(A_{n, \mathrm{DCI}}\right)= & :-\frac{\gamma_{c}(a)}{8} A_{n, \mathrm{DCI}}^{(1)}+\frac{B_{\delta}(a)}{2}\left[n \log (\delta)+n+\frac{1}{2} \log \left(w_{1} \cdots w_{n}\right)\right] \\
& +n \widehat{f}(a)+\widehat{C}(a)+R_{n}(a)
\end{aligned}
$$

where $B_{\delta}(a):=3 \zeta_{3} a^{2}+\mathcal{O}\left(a^{3}\right)$ is the so-called virtual anomalous dimension [90, 91], and the functions $\widehat{f}(a)$ and $\widehat{C}(a)$ are analogous to $\widetilde{f}(a)$ and $\widetilde{C}(a)$ - which could not be disentangled from each other knowing the logarithm for six particles alone.

In [71], the six-point logarithm was shown to take the form ${ }^{6}$

$$
\log \left(A_{6, \mathrm{DCI}}\right)^{(2)}=-\zeta_{2} A_{6, \mathrm{DCI}}^{(1)}+\frac{3}{2} \zeta_{3}\left[6 \log (\delta)+6+\frac{1}{2} \log \left(w_{1} \cdots w_{6}\right)\right]-\frac{49 \pi^{4}}{720}+R_{6}^{(2)} ;
$$

and for five particles, starting from representation given in (2.6), it is not hard to show that $^{7}$

$$
\log \left(A_{5, \mathrm{DCI}}\right)^{(2)}=-\zeta_{2} A_{5, \mathrm{DCI}}^{(1)}+\frac{3}{2} \zeta_{3}\left[5 \log (\delta)+5+\log \left(w_{1} \cdots w_{5}\right)\right]-\frac{17 \pi^{4}}{288}+R_{5}^{(2)} .
$$

Combining this with our new result for seven particles,

$$
\log \left(A_{7, \mathrm{DCI}}\right)^{(2)}=-\zeta_{2} A_{7, \mathrm{DCI}}^{(1)}+\frac{3}{2} \zeta_{3}\left[7 \log (\delta)+7+\frac{1}{2} \log \left(w_{1} \cdots w_{7}\right)\right]-\frac{37 \pi^{4}}{480}+R_{7}^{(2)},
$$

allows us to conclude that, in the conformal regularization scheme,

$$
\widehat{f}(a)=-\frac{1}{2}\left(\zeta_{4}+\frac{1}{4} \zeta_{2}^{2}\right) a^{2}+\mathcal{O}\left(a^{3}\right), \quad \widehat{C}(a)=-\frac{1}{2} \zeta_{2}^{2} a^{2}+\mathcal{O}\left(a^{3}\right) .
$$

Although already mentioned in the introduction, it is worth pausing to note that, in the representation of the logarithm (4.15), the remainder function $R_{7}^{(2)}$ numerically matches the analytic expression derived in [70] from the symbol (from [67]).

\subsection{Symbology and the alphabets of individual integral contributions}

Interestingly, almost all of the seed integrals we compute contain symbol letters that are not present in the full remainder function. The integral $I_{1}$ is the only exception: it in fact requires only the ordinary hexagon-function symbol alphabet. However, each of the other integrals involve spurious (but rational) symbol letters. Specifically, each of $\left\{I_{2}, I_{3}, I_{4}\right\}$ involve two 'new' letters relative to the remainder function, and $I_{5}$ involves nine additional letters (after all the simplifications described in subsection 3.3). In cyclic sum, however, all these additional letters cancel - and quite nontrivially. For example, among these contributions only the entire cyclic sum of $\left(I_{2}+I_{3}+I_{4}+I_{5}\right)$ is free of 'spurious' letters relative to the 42 letter alphabet expected for heptagon functions [70] (see also [47-49, 92-96]). For the sake of those readers interested in more details, we have provided the additional symbol letters that arise for the cyclic seed integrals in the supplementary material attached to this work.

\footnotetext{
${ }^{6}$ Nota bene: for six particles, $\left(w_{1} \cdots w_{6}\right)=\left(w_{1} w_{2} w_{3}\right)^{2}$, with $w_{i}$ more familiarly denoted $\{u, v, w\}$.

${ }^{7}$ Nota bene: for five particles, $w_{a}=1$ for all $a$ and $R_{5}^{(\ell)}=0$ for all $\ell$.
} 


\section{Discussion}

In this paper, we have computed the logarithm of the two-loop MHV amplitude at seven points in planar, maximally supersymmetric $(\mathcal{N}=4)$ super Yang-Mills theory directly from a local integrand representation. In doing so, we have shown that carefully preserving the symmetries of the theory makes computations dramatically easier, even when using otherwise traditional methods. However, these methods are still not optimal: as we have seen, issues of linear reducibility make some of the integrals we find unsuitable for expansion into a fibration basis (by known methods), resulting in a sometimes unnecessarily-spurious symbol alphabet. It would be interesting to see whether other common methods (for example, differential equations, or integration-by-parts reduction) can simplify this calculation further.

In using the dual conformal regularization of [71], we have checked the conjectures for the scheme dependence of the logarithm of the amplitude put forward in that paper. It would be interesting to check these conjectures at higher loop orders, and more generally, to understand in detail the relationship between the conformal regulator and the Higgs regulator.

\section{Acknowledgments}

This work was supported in part by the Danish National Research Foundation (DNRF91), an ERC Starting Grant (No. 757978), a grant from the Villum Fonden (No. 15369), and the European Union's Horizon 2020 research and innovation program under grant agreement No. $793151(\mathrm{MvH})$. We are grateful for the hospitality of the Harvard Center for Mathematical Sciences and Applications, and JLB and MvH would like to thank the Aspen Center for Physics, which is supported by National Science Foundation grant PHY-1607611.

Open Access. This article is distributed under the terms of the Creative Commons Attribution License (CC-BY 4.0), which permits any use, distribution and reproduction in any medium, provided the original author(s) and source are credited.

\section{References}

[1] N. Arkani-Hamed, F. Cachazo, C. Cheung and J. Kaplan, A Duality For The S Matrix, JHEP 03 (2010) 020 [arXiv: 0907.5418] [INSPIRE].

[2] N. Arkani-Hamed, J. Bourjaily, F. Cachazo and J. Trnka, Unification of Residues and Grassmannian Dualities, JHEP 01 (2011) 049 [arXiv:0912.4912] [INSPIRE].

[3] N. Arkani-Hamed, J.L. Bourjaily, F. Cachazo, A.B. Goncharov, A. Postnikov and J. Trnka, Grassmannian Geometry of Scattering Amplitudes, Cambridge University Press (2016) [arXiv:1212.5605] [INSPIRE].

[4] N. Arkani-Hamed and J. Trnka, The Amplituhedron, JHEP 10 (2014) 030 [arXiv: 1312.2007] [INSPIRE].

[5] C. Frye, H. Hannesdottir, N. Paul, M.D. Schwartz and K. Yan, Infrared Finiteness and Forward Scattering, Phys. Rev. D 99 (2019) 056015 [arXiv: 1810.10022] [INSPIRE].

[6] H. Hannesdottir and M.D. Schwartz, A Finite S-matrix, arXiv:1906.03271 [INSPIRE]. 
[7] N. Arkani-Hamed, J.L. Bourjaily, F. Cachazo, S. Caron-Huot and J. Trnka, The All-Loop Integrand For Scattering Amplitudes in Planar $\mathcal{N}=4$ SYM, JHEP 01 (2011) 041 [arXiv: 1008.2958] [INSPIRE].

[8] P. Benincasa, On-shell diagrammatics and the perturbative structure of planar gauge theories, arXiv: 1510.03642 [INSPIRE].

[9] J.L. Bourjaily, A. DiRe, A. Shaikh, M. Spradlin and A. Volovich, The Soft-Collinear Bootstrap: $\mathcal{N}=4$ Yang-Mills Amplitudes at Six and Seven Loops, JHEP 03 (2012) 032 [arXiv: 1112.6432] [INSPIRE].

[10] J.L. Bourjaily, P. Heslop and V.-V. Tran, Perturbation Theory at Eight Loops: Novel Structures and the Breakdown of Manifest Conformality in $\mathcal{N}=4$ Supersymmetric Yang-Mills Theory, Phys. Rev. Lett. 116 (2016) 191602 [arXiv:1512.07912] [INSPIRE].

[11] J.L. Bourjaily, P. Heslop and V.-V. Tran, Amplitudes and Correlators to Ten Loops Using Simple, Graphical Bootstraps, JHEP 11 (2016) 125 [arXiv:1609.00007] [INSPIRE].

[12] C. Baadsgaard, N.E.J. Bjerrum-Bohr, J.L. Bourjaily, S. Caron-Huot, P.H. Damgaard and B. Feng, New Representations of the Perturbative S-matrix, Phys. Rev. Lett. 116 (2016) 061601 [arXiv: 1509.02169] [INSPIRE].

[13] Z. Bern, L.J. Dixon, D.C. Dunbar and D.A. Kosower, One loop n-point gauge theory amplitudes, unitarity and collinear limits, Nucl. Phys. B 425 (1994) 217 [hep-ph/9403226] [INSPIRE].

[14] Z. Bern, L.J. Dixon, D.C. Dunbar and D.A. Kosower, Fusing gauge theory tree amplitudes into loop amplitudes, Nucl. Phys. B 435 (1995) 59 [hep-ph/9409265] [INSPIRE].

[15] R. Britto, F. Cachazo and B. Feng, Generalized unitarity and one-loop amplitudes in $\mathcal{N}=4$ super-Yang-Mills, Nucl. Phys. B 725 (2005) 275 [hep-th/0412103] [INSPIRE].

[16] Z. Bern, M. Czakon, L.J. Dixon, D.A. Kosower and V.A. Smirnov, The Four-Loop Planar Amplitude and Cusp Anomalous Dimension in Maximally Supersymmetric Yang-Mills Theory, Phys. Rev. D 75 (2007) 085010 [hep-th/0610248] [InSPIRE].

[17] C. Anastasiou, R. Britto, B. Feng, Z. Kunszt and P. Mastrolia, D-dimensional unitarity cut method, Phys. Lett. B 645 (2007) 213 [hep-ph/0609191] [INSPIRE].

[18] Z. Bern, J.J.M. Carrasco, H. Johansson and D.A. Kosower, Maximally supersymmetric planar Yang-Mills amplitudes at five loops, Phys. Rev. D 76 (2007) 125020 [arXiv: 0705.1864] [INSPIRE].

[19] F. Cachazo, Sharpening The Leading Singularity, arXiv:0803.1988 [INSPIRE].

[20] Z. Bern et al., The Two-Loop Six-Gluon MHV Amplitude in Maximally Supersymmetric Yang-Mills Theory, Phys. Rev. D 78 (2008) 045007 [arXiv:0803.1465] [INSPIRE].

[21] C.F. Berger et al., An Automated Implementation of On-Shell Methods for One-Loop Amplitudes, Phys. Rev. D 78 (2008) 036003 [arXiv:0803.4180] [INSPIRE].

[22] S. Abreu, F. Febres Cordero, H. Ita, M. Jaquier, B. Page and M. Zeng, Two-Loop Four-Gluon Amplitudes from Numerical Unitarity, Phys. Rev. Lett. 119 (2017) 142001 [arXiv: 1703.05273] [INSPIRE].

[23] N. Arkani-Hamed, J.L. Bourjaily, F. Cachazo and J. Trnka, Local Integrals for Planar Scattering Amplitudes, JHEP 06 (2012) 125 [arXiv: 1012.6032] [INSPIRE]. 
[24] J.L. Bourjaily, S. Caron-Huot and J. Trnka, Dual-Conformal Regularization of Infrared Loop Divergences and the Chiral Box Expansion, JHEP 01 (2015) 001 [arXiv:1303.4734] [INSPIRE].

[25] J.L. Bourjaily and J. Trnka, Local Integrand Representations of All Two-Loop Amplitudes in Planar SYM, JHEP 08 (2015) 119 [arXiv: 1505.05886] [INSPIRE].

[26] J.L. Bourjaily, E. Herrmann and J. Trnka, Prescriptive Unitarity, JHEP 06 (2017) 059 [arXiv: 1704.05460] [INSPIRE].

[27] J.L. Bourjaily, E. Herrmann and J. Trnka, Maximally supersymmetric amplitudes at infinite loop momentum, Phys. Rev. D 99 (2019) 066006 [arXiv:1812.11185] [INSPIRE].

[28] J.L. Bourjaily, E. Herrmann, C. Langer, A.J. McLeod and J. Trnka, Prescriptive Unitarity for Non-Planar Six-Particle Amplitudes at Two Loops, JHEP 12 (2019) 073 [arXiv: 1909.09131] [INSPIRE].

[29] J.L. Bourjaily, E. Herrmann, C. Langer, A.J. McLeod and J. Trnka, All-Multiplicity Non-Planar MHV Amplitudes in SYM at Two Loops, arXiv:1911.09106 [INSPIRE].

[30] S. Mandelstam, Light Cone Superspace and the Ultraviolet Finiteness of the $\mathcal{N}=4$ Model, Nucl. Phys. B 213 (1983) 149 [inSPIRE].

[31] L. Brink, O. Lindgren and B.E.W. Nilsson, The Ultraviolet Finiteness of the $\mathcal{N}=4$ Yang-Mills Theory, Phys. Lett. 123B (1983) 323 [INSPIRE].

[32] P.S. Howe, K.S. Stelle and P.K. Townsend, Miraculous Ultraviolet Cancellations in Supersymmetry Made Manifest, Nucl. Phys. B 236 (1984) 125 [INSPIRE].

[33] J.M. Drummond, J. Henn, V.A. Smirnov and E. Sokatchev, Magic identities for conformal four-point integrals, JHEP 01 (2007) 064 [hep-th/0607160] [INSPIRE].

[34] L.F. Alday and J.M. Maldacena, Gluon scattering amplitudes at strong coupling, JHEP 06 (2007) 064 [arXiv:0705.0303] [INSPIRE].

[35] J.M. Drummond, J. Henn, G.P. Korchemsky and E. Sokatchev, Dual superconformal symmetry of scattering amplitudes in $N=4$ super-Yang-Mills theory, Nucl. Phys. B 828 (2010) 317 [arXiv:0807.1095] [inSPIRE].

[36] V. Del Duca, C. Duhr and V.A. Smirnov, The Two-Loop Hexagon Wilson Loop in $\mathcal{N}=4$ SYM, JHEP 05 (2010) 084 [arXiv:1003.1702] [INSPIRE].

[37] A.B. Goncharov, M. Spradlin, C. Vergu and A. Volovich, Classical Polylogarithms for Amplitudes and Wilson Loops, Phys. Rev. Lett. 105 (2010) 151605 [arXiv:1006.5703] [INSPIRE].

[38] L.J. Dixon, J.M. Drummond and J.M. Henn, Analytic result for the two-loop six-point NMHV amplitude in $N=4$ super Yang-Mills theory, JHEP 01 (2012) 024 [arXiv: 1111.1704] [INSPIRE].

[39] L.J. Dixon, J.M. Drummond, M. von Hippel and J. Pennington, Hexagon functions and the three-loop remainder function, JHEP 12 (2013) 049 [arXiv:1308.2276] [INSPIRE].

[40] L.J. Dixon, J.M. Drummond, C. Duhr and J. Pennington, The four-loop remainder function and multi-Regge behavior at NNLLA in planar $\mathcal{N}=4$ super-Yang-Mills theory, JHEP 06 (2014) 116 [arXiv:1402.3300] [INSPIRE].

[41] L.J. Dixon and M. von Hippel, Bootstrapping an NMHV amplitude through three loops, JHEP 10 (2014) 065 [arXiv: 1408.1505] [InSPIRE]. 
[42] L.J. Dixon, M. von Hippel and A.J. McLeod, The four-loop six-gluon NMHV ratio function, JHEP 01 (2016) 053 [arXiv: 1509.08127] [INSPIRE].

[43] L.J. Dixon, M. von Hippel, A.J. McLeod and J. Trnka, Multi-loop positivity of the planar $\mathcal{N}=4$ SYM six-point amplitude, JHEP 02 (2017) 112 [arXiv:1611.08325] [INSPIRE].

[44] S. Caron-Huot, L.J. Dixon, A. McLeod and M. von Hippel, Bootstrapping a Five-Loop Amplitude Using Steinmann Relations, Phys. Rev. Lett. 117 (2016) 241601 [arXiv: 1609.00669] [INSPIRE].

[45] S. Caron-Huot, L.J. Dixon, F. Dulat, M. von Hippel, A.J. McLeod and G. Papathanasiou, Six-Gluon amplitudes in planar $\mathcal{N}=4$ super-Yang-Mills theory at six and seven loops, JHEP 08 (2019) 016 [arXiv: 1903.10890] [INSPIRE].

[46] S. Caron-Huot, L.J. Dixon, F. Dulat, M. Von Hippel, A.J. McLeod and G. Papathanasiou, The Cosmic Galois Group and Extended Steinmann Relations for Planar $\mathcal{N}=4 S Y M$ Amplitudes, JHEP 09 (2019) 061 [arXiv:1906.07116] [inSPIRE].

[47] J.M. Drummond, G. Papathanasiou and M. Spradlin, A Symbol of Uniqueness: The Cluster Bootstrap for the 3-Loop MHV Heptagon, JHEP 03 (2015) 072 [arXiv: 1412.3763] [INSPIRE].

[48] L.J. Dixon, J. Drummond, T. Harrington, A.J. McLeod, G. Papathanasiou and M. Spradlin, Heptagons from the Steinmann Cluster Bootstrap, JHEP 02 (2017) 137 [arXiv:1612.08976] [INSPIRE].

[49] J. Drummond, J. Foster, Ö. Gürdoğan and G. Papathanasiou, Cluster adjacency and the four-loop NMHV heptagon, JHEP 03 (2019) 087 [arXiv: 1812.04640] [INSPIRE].

[50] S. Abreu, L.J. Dixon, E. Herrmann, B. Page and M. Zeng, The two-loop five-point amplitude in $\mathcal{N}=4$ super-Yang-Mills theory, Phys. Rev. Lett. 122 (2019) 121603 [arXiv:1812.08941] [INSPIRE].

[51] D. Chicherin, T. Gehrmann, J.M. Henn, P. Wasser, Y. Zhang and S. Zoia, Analytic result for a two-loop five-particle amplitude, Phys. Rev. Lett. 122 (2019) 121602 [arXiv:1812.11057] [INSPIRE].

[52] T. Gehrmann, J.M. Henn and N.A. Lo Presti, Analytic form of the two-loop planar five-gluon all-plus-helicity amplitude in QCD, Phys. Rev. Lett. 116 (2016) 062001 [Erratum ibid. 116 (2016) 189903] [arXiv: 1511.05409] [INSPIRE].

[53] S. Badger, C. Brønnum-Hansen, H.B. Hartanto and T. Peraro, First look at two-loop five-gluon scattering in QCD, Phys. Rev. Lett. 120 (2018) 092001 [arXiv:1712.02229] [INSPIRE].

[54] S. Abreu, F. Febres Cordero, H. Ita, B. Page and M. Zeng, Planar Two-Loop Five-Gluon Amplitudes from Numerical Unitarity, Phys. Rev. D 97 (2018) 116014 [arXiv:1712.03946] [INSPIRE].

[55] S. Abreu, F. Febres Cordero, H. Ita, B. Page and V. Sotnikov, Planar Two-Loop Five-Parton Amplitudes from Numerical Unitarity, JHEP 11 (2018) 116 [arXiv:1809.09067] [InSPIRE].

[56] S. Badger, C. Brønnum-Hansen, H.B. Hartanto and T. Peraro, Analytic helicity amplitudes for two-loop five-gluon scattering: the single-minus case, JHEP 01 (2019) 186 [arXiv: 1811.11699] [INSPIRE].

[57] S. Abreu, J. Dormans, F. Febres Cordero, H. Ita and B. Page, Analytic Form of Planar Two-Loop Five-Gluon Scattering Amplitudes in QCD, Phys. Rev. Lett. 122 (2019) 082002 [arXiv: 1812.04586] [INSPIRE]. 
[58] D. Chicherin, T. Gehrmann, J.M. Henn, P. Wasser, Y. Zhang and S. Zoia, All Master Integrals for Three-Jet Production at Next-to-Next-to-Leading Order, Phys. Rev. Lett. 123 (2019) 041603 [arXiv: 1812.11160] [INSPIRE].

[59] D. Chicherin, T. Gehrmann, J.M. Henn, P. Wasser, Y. Zhang and S. Zoia, The two-loop five-particle amplitude in $\mathcal{N}=8$ supergravity, JHEP 03 (2019) 115 [arXiv:1901.05932] [INSPIRE].

[60] S. Abreu, L.J. Dixon, E. Herrmann, B. Page and M. Zeng, The two-loop five-point amplitude in $\mathcal{N}=8$ supergravity, JHEP 03 (2019) 123 [arXiv:1901.08563] [INSPIRE].

[61] J.L. Bourjaily, A.J. McLeod, M. von Hippel and M. Wilhelm, Bounded Collection of Feynman Integral Calabi-Yau Geometries, Phys. Rev. Lett. 122 (2019) 031601 [arXiv: 1810.07689] [INSPIRE].

[62] F.C.S. Brown, On the periods of some Feynman integrals, arXiv:0910.0114 [INSPIRE].

[63] E. Panzer, Algorithms for the symbolic integration of hyperlogarithms with applications to Feynman integrals, Comput. Phys. Commun. 188 (2015) 148 [arXiv:1403.3385] [InSPIRE].

[64] HyperInt, https://bitbucket.org/PanzerErik/hyperint/wiki/Home.

[65] J.L. Bourjaily, A.J. McLeod, M. von Hippel and M. Wilhelm, Rationalizing Loop Integration, JHEP 08 (2018) 184 [arXiv: 1805.10281] [INSPIRE].

[66] J.L. Bourjaily, A.J. McLeod, C. Vergu, M. Volk, M. Von Hippel and M. Wilhelm, Rooting Out Letters: Octagonal Symbol Alphabets and Algebraic Number Theory, JHEP 02 (2020) 025 [arXiv: 1910.14224] [INSPIRE].

[67] S. Caron-Huot, Superconformal symmetry and two-loop amplitudes in planar $\mathcal{N}=4$ super Yang-Mills, JHEP 12 (2011) 066 [arXiv:1105.5606] [INSPIRE].

[68] S. Caron-Huot, Notes on the scattering amplitude/Wilson loop duality, JHEP 07 (2011) 058 [arXiv: 1010.1167] [INSPIRE].

[69] S. Caron-Huot and S. He, Jumpstarting the All-Loop S-matrix of Planar $\mathcal{N}=4$ Super Yang-Mills, JHEP 07 (2012) 174 [arXiv:1112.1060] [INSPIRE].

[70] J. Golden and M. Spradlin, An analytic result for the two-loop seven-point MHV amplitude in $\mathcal{N}=4$ SYM, JHEP 08 (2014) 154 [arXiv:1406.2055] [INSPIRE].

[71] J.L. Bourjaily, F. Dulat and E. Panzer, Manifestly Dual-Conformal Loop Integration, Nucl. Phys. B 942 (2019) 251 [arXiv: 1901.02887] [InSPIRE].

[72] C. Vergu, Higher point $M H V$ amplitudes in $\mathcal{N}=4$ Supersymmetric Yang-Mills Theory, Phys. Rev. D 79 (2009) 125005 [arXiv:0903.3526] [INSPIRE].

[73] C. Vergu, The Two-loop MHV amplitudes in $\mathcal{N}=4$ supersymmetric Yang-Mills theory, Phys. Rev. D 80 (2009) 125025 [arXiv:0908.2394] [INSPIRE].

[74] A. Hodges, Eliminating spurious poles from gauge-theoretic amplitudes, JHEP 05 (2013) 135 [arXiv: 0905.1473] [INSPIRE].

[75] H. Cheng and T.T. Wu, Expanding Protons: Scattering at High Energies, Cambridge, U.S.A., MIT-PR. (1987) [INSPIRE].

[76] J.L. Bourjaily, Positroids, Plabic Graphs and Scattering Amplitudes in Mathematica, arXiv:1212.6974 [INSPIRE].

[77] C. Anastasiou, C. Duhr, F. Dulat and B. Mistlberger, Soft triple-real radiation for Higgs production at $N_{3} L O, J H E P 07$ (2013) 003 [arXiv: 1302.4379] [INSPIRE]. 
[78] E. Panzer, On hyperlogarithms and Feynman integrals with divergences and many scales, JHEP 03 (2014) 071 [arXiv: 1401.4361] [INSPIRE].

[79] F. Brown, The Massless higher-loop two-point function, Commun. Math. Phys. 287 (2009) 925 [arXiv:0804.1660] [INSPIRE].

[80] M. Besier, D. Van Straten and S. Weinzierl, Rationalizing roots: an algorithmic approach, Commun. Num. Theor. Phys. 13 (2019) 253 [arXiv:1809.10983] [INSPIRE].

[81] N. Arkani-Hamed, J.L. Bourjaily, F. Cachazo and J. Trnka, Singularity Structure of Maximally Supersymmetric Scattering Amplitudes, Phys. Rev. Lett. 113 (2014) 261603 [arXiv: 1410.0354] [INSPIRE].

[82] C. Duhr, H. Gangl and J.R. Rhodes, From polygons and symbols to polylogarithmic functions, JHEP 10 (2012) 075 [arXiv:1110.0458] [INSPIRE].

[83] L.F. Alday, J.M. Henn, J. Plefka and T. Schuster, Scattering into the fifth dimension of $\mathcal{N}=4$ super Yang-Mills, JHEP 01 (2010) 077 [arXiv:0908.0684] [INSPIRE].

[84] J.M. Henn, S.G. Naculich, H.J. Schnitzer and M. Spradlin, More loops and legs in Higgs-regulated $\mathcal{N}=4$ SYM amplitudes, JHEP 08 (2010) 002 [arXiv:1004.5381] [INSPIRE].

[85] Z. Bern, L.J. Dixon and V.A. Smirnov, Iteration of planar amplitudes in maximally supersymmetric Yang-Mills theory at three loops and beyond, Phys. Rev. D 72 (2005) 085001 [hep-th/0505205] [INSPIRE].

[86] G.F. Sterman and M.E. Tejeda-Yeomans, Multiloop amplitudes and resummation, Phys. Lett. B 552 (2003) 48 [hep-ph/0210130] [INSPIRE].

[87] N. Beisert, B. Eden and M. Staudacher, Transcendentality and Crossing, J. Stat. Mech. 0701 (2007) P01021 [hep-th/0610251] [INSPIRE].

[88] D. Correa, J. Henn, J. Maldacena and A. Sever, The cusp anomalous dimension at three loops and beyond, JHEP 05 (2012) 098 [arXiv:1203.1019] [INSPIRE].

[89] J.M. Henn and T. Huber, The four-loop cusp anomalous dimension in $\mathcal{N}=4$ super Yang-Mills and analytic integration techniques for Wilson line integrals, JHEP 09 (2013) 147 [arXiv: 1304.6418] [INSPIRE].

[90] A.V. Kotikov, L.N. Lipatov, A.I. Onishchenko and V.N. Velizhanin, Three loop universal anomalous dimension of the Wilson operators in $\mathcal{N}=4$ SUSY Yang-Mills model, Phys. Lett. B 595 (2004) 521 [Erratum ibid. B 632 (2006) 754] [hep-th/0404092] [INSPIRE].

[91] L. Freyhult, A. Rej and M. Staudacher, A Generalized Scaling Function for AdS/CFT, J. Stat. Mech. 0807 (2008) P07015 [arXiv:0712.2743] [INSPIRE].

[92] J. Golden and M. Spradlin, The differential of all two-loop MHV amplitudes in $\mathcal{N}=4$ Yang-Mills theory, JHEP 09 (2013) 111 [arXiv:1306.1833] [INSPIRE].

[93] J. Golden, A.B. Goncharov, M. Spradlin, C. Vergu and A. Volovich, Motivic Amplitudes and Cluster Coordinates, JHEP 01 (2014) 091 [arXiv: 1305.1617] [INSPIRE].

[94] J. Golden and M. Spradlin, A Cluster Bootstrap for Two-Loop MHV Amplitudes, JHEP 02 (2015) 002 [arXiv:1411.3289] [INSPIRE].

[95] J. Golden, M.F. Paulos, M. Spradlin and A. Volovich, Cluster Polylogarithms for Scattering Amplitudes, J. Phys. A 47 (2014) 474005 [arXiv:1401.6446] [InSPIRE].

[96] J. Drummond, J. Foster and Ö. Gürdoğan, Cluster Adjacency Properties of Scattering Amplitudes in $\mathcal{N}=4$ Supersymmetric Yang-Mills Theory, Phys. Rev. Lett. 120 (2018) 161601 [arXiv: 1710.10953] [INSPIRE]. 